MS061.002

Microsymposium

\title{
Magnetic frustration and random exchange in double perovskites
}

Sami Vasala ${ }^{1}$, Otto Mustonen ${ }^{2}$, Maarit Karppinen $^{2}$, Elisa Baggio-Saitovitch ${ }^{1}$

${ }^{1}$ Centro Brasileiro De Pesquisas Físicas, Rio De Janeiro, Brazil, ${ }^{2}$ Department of Chemistry, Aalto University, Espoo, Finland E-mail: svasala@gmail.com

The double-perovskite structure offers an exciting platform for studying novel magnetic properties. Here I present our recent results on the B-site ordered double perovskites $\mathrm{Sr} 2 \mathrm{Cu}(\mathrm{Te}, \mathrm{W}) \mathrm{O6}$, which show low-dimensional magnetic properties and possible spin liquid -type behavior. The structure of these materials is three dimensional, but Jahn-Teller distortion and a subsequent orbital ordering results in an effective square lattice of the $S=1 / 2 \mathrm{Cu} 2+$ ions.

The magnetic ground state in a square lattice depends on the ratio of the nearest neighbor (NN) and next-nearest neighbor (NNN) interactions, and with frustrated interactions, novel ground states such as spin liquids may be obtained. In $\mathrm{Sr} 2 \mathrm{Cu}(\mathrm{Te}, \mathrm{W}) \mathrm{O6}$, the magnetic interaction are mediated through the O-Te/W-O bonds, and the choice of the second $\mathrm{B}-\mathrm{site}$ cation dictates the magnetic properties: Te causes the NN interactions to be the strongest, while W results in NNN interactions dominating. Partial cation substitution leads to a random arrangement of Te and $W$ on the second B-site, which results in random exchange pathways between the $\mathrm{Cu}$ ions. In the solid solution Sr2CuTe1-xWxO6, the combination of frustration and randomness of the magnetic interactions causes a complete suppression of long-range magnetic order.

The implications of the results on other double perovskite compounds are also discussed. It is a common feature in the A2B'B"O6 double-perovskite compounds that the choice of the non-magnetic B"-site cation can affect the magnetic interactions between the magnetic $B^{\prime}$ cations. This behavior combined with the random exchanges caused by a partial $B^{\prime \prime}-$ site cation substitution is expected to result in many more interesting materials with suppressed magnetic ordering and novel ground states.

Keywords: double perovskites, magnetic frustration, random exchange 